\title{
Surveillance cardiac catheterisation in heart transplant recipients
}

With continued improvements in immunosuppressive therapy and management of rejection, the principal problem limiting long term survival of patients following heart transplantation has been the development of cardiac allograft vasculopathy (CAV). Virtually all patients who survive beyond one year have vascular changes of CAV with the extent and severity of involvement correlating with patient survival. ${ }^{1}$ The lack of consistent clinical signs and symptoms of CAV, the lack of reliable non-invasive testing, and the rapidity of progression with poor prognosis, have convinced most centres to perform annual coronary angiograms post-transplantation as a matter of policy.

Unlike typical atherosclerotic coronary disease with discernable risk factors, there are no clear clinical, immunological or epidemiological factors that identify the transplant recipients at risk of developing severe CAV. Cardiac ischaemia caused by CAV does not typically present as angina pectoris but rather as myocardial infarction, sudden death or congestive heart failure secondary to impaired ventricular function. Chest pain in a transplant patient is usually not indicative of ischaemia; moreover, ischaemia typically occurs in the absence of significant chest discomfort. Acute myocardial infarction is often silent or presents with atypical symptomatology. In a review of transplant patients experiencing acute myocardial infarction, chest pain was not a prominent feature, ECG changes were non-specific, and infarct related mortality approached $30 \%$. All patients who suffered an acute myocardial infarction were known to have had significant CAV from their annual coronary angiographic studies. ${ }^{2}$

The practice of routine coronary angiography has been supported by the lack of other effective means of monitoring disease progression. Given the diffuse nature of $\mathrm{CAV}$, involvement of the microvascular as well as the epicardial vessels, nuclear medicine studies predicated on regional perfusion variations have been unreliable for detection of ischaemia. In a prospective study evaluating echocardiography, rest and exercise gated wall motion studies, ambulatory ECG monitoring, exercise ECG and persantinethallium studies, the predictive values for all modalities in detecting CAV were quite low. Echocardiographic detection of segmental wall motion abnormalities had the highest sensitivity at $58 \% .^{3}$ Stress echocardiographic techniques are currently being evaluated and appear promising, but additional large scale trials need to be performed. ${ }^{4}$

Coronary angiography demonstrates characteristic differences distinguishing CAV as a separate entity from typical atherosclerotic disease. The presence of angiographically documented disease occurs in $\sim 50 \%$ of patients five years after transplantation. In addition to typical proximal stenoses of the epicardial vessels, CAV commonly exhibits diffuse, concentric involvement of the secondary and tertiary branches. This distal "pruning" of the vasculature often is not appreciated until careful comparisons have been made between serial studies. The shortcomings of angiography for the detection and monitoring of CAV have become apparent in recent years. The introduction of intravascular ultrasonography (IVUS) has shown that early and extensive plaque development can occur without significant lumenal encroachment or angiographic evidence of disease. ${ }^{6}$ IVUS has further demonstrated that accelerated development of disease occurs in the first two years following transplantation with continued but less aggressive progression in subsequent years. ${ }^{78}$

In addition to structural information obtained from coronary angiography and intravascular ultrasound studies, physiological information including the integrity of endothelial function and assessment of coronary vasodilatory reserve can be acquired during surveillance cardiac catheterisation. Endothelial dysfunction can occur early post-transplantation, even in the absence of angiographically detectable CAV. ${ }^{9}$ Conversely, preserved endothelial function, even in the presence of significant CAV, has been demonstrated in long term transplant survivors and may identify patients with better long term prognosis. ${ }^{10}$ Measurement of coronary vasodilatory reserve using new Doppler and pressure coronary wires may enable us to assess the impact of CAV on the microvasculature and further define long term risk for individual patients. ${ }^{11}$ While the practical applicability of these assessments remains to be seen, this information continues to enhance our understanding of the disease process.

The inability to understand fully the pathophysiology of CAV has significantly hampered attempts to ameliorate its consequences and improve transplant recipient outcomes. However, in spite of our incomplete understanding of CAV risk factors, some progress has been made. For example, hyperlipidaemia following cardiac transplantation is common although a clear causal association with the development of CAV has never been established definitively. Concerns regarding the interaction of HMGCoA reductase inhibitors with cyclosporin-that is, potential rhabdomyolysis, have made physicians reluctant to use these agents in the transplant population. Two recent studies, however, have shown a trend towards less CAV and potential improved survival in patients treated with simvistatin $^{12}$ or pravastatin. ${ }^{13} \mathrm{New}$ immunosuppressive agents such as rapamycin and mycophenolate mofitil are being studied carefully for potential secondary benefits in retarding the development of CAV. Drugs that enhance endothelial function are also being studied and may have a beneficial effect on graft survival. Identification of patients with early evidence of CAV could help the patients most likely to benefit from new treatments.

Given the lack of symptoms and absence of accurate detection of ischaemia by non-invasive means, most coronary interventions for CAV have been performed because of the presence of severe angiographic disease. Given the diffuse nature of the process, the number of patients with focal stenóses amenable to percutaneous strategies represents a distinct minority of all patients with CAV. Although procedural success rates have been high and comparable to patients with atherosclerosis, long term outcomes have not been very satisfactory with high rates of restenosis and minimal discernable impact on survival. ${ }^{14}$ Experience with coronary artery stenting has yet to be 
reported but the likelihood of significant improvement over traditional interventional techniques is low. Given the poor outcome associated with CAV and lack of other alternatives, retransplantation has been offered as a therapeutic option when the disease limits the likelihood of long term survival. Survival rates after retransplantation are poor compared with primary transplantation, but a number of patients have done well; CAV as the indication for retransplantation has been associated with better outcomes. ${ }^{15}$

As our experience and understanding of CAV has matured, many physicians have begun to question the usefulness of routine coronary angiography in this patient population. Studies have demonstrated that disease severity varies tremendously within the transplant population. There is clearly a group of patients who develop an aggressive form of disease within months of transplantation and another group that follows a much more benign course, demonstrating minimal disease even many years after transplantation. There are currently no predictors that stratify risk of CAV, but identification of patient subtypes may become feasible using information obtained from cardiac catheterisation including coronary angiography, IVUS, endothelial function assessment, and determination of vasomotor competency. Patients with favourable profiles and minimal disease progression could be permitted to undergo less stringent evaluation; patients with rapid progression would receive more attentive follow up and could be considered for more aggressive treatment, including novel pharmacological, immunological, and interventional strategies.

At Stanford, we have continued to perform annual cardiac catheterisation in our transplant recipients with a high percentage of patients participating in ongoing or new clinical trials. As CAV remains the principal impediment to patient longevity, we feel compelled to monitor its progression even if current therapeutic options are less than ideal. The decision to forego periodic assessment of CAV is made on an individual basis when there is patient and physician consensus that the information obtained would not be beneficial or the risks of the procedure appear unacceptably high. Patients with severe CAV who are acceptable retransplant candidates have been provided with this option. Despite suboptimal long term outcomes with percutaneous interventions, they have been offered to patients as palliative therapy. These policies reflect our long term commitment to the patient from the initial deci- sion to pursue transplantation as therapy for their end stage heart disease. We believe that continued investigative endeavours performed in conjunction with routine surveillance examinations will lead to improved treatment strategies for CAV.

Stanford University School of Medicine, CHARLES B KIM ALAN C YEUNG

Stanford, CA 94305, USA

1 Keogh AM, Valantine HA, Hunt SA, Schroeder JS, McIntosh N, Oyer PE, et al. Impact of proximal or midvessel discrete coronary artery stenosis on survival after heart transplantation. If Heart Lung Transplant

2 Gao SZ, Schroeder JS, Hunt SA, Billingham ME, Valantine HA, Stinson EB. Acute myocardial infarction in cardiac transplant recipients. $\mathrm{Am} \mathcal{F}$ Cardiol 1989;64:1093-7.

3 Smart FW, Ballantyne CM, Cocanougher B, Farmer JA, Sokola ME, Noon GP, et al. Insensitivity of noninvasive tests to detect coronary artery vasculopathy after heart transplant. Am $\mathcal{\text { Cardiol }} 1991 ; 67: 243-7$.

4 Collings CA, Pinto FJ, Valantine HA, Popylisen S, Puryear JV, Schnittger I. Exercise echocardiography in heart transplant recipients: a comparison with angiography and intracoronary ultrasonography. F Heart Lung with angiography and intracc

5 Uretsky BJ, Murali S, Reddy PS, Rabin B, Lee A, Griffith BP, et al. Development of coronary artery disease in cardiac transplant patients Development of coronary artery disease in cardiac transplant patients
receiving immunosuppressive therapy with cyclosporine and prednisone. receiving immunosuppressive

6 St Goar FD, Pinto FJ, Alderman EL, Valantine HA, Schroeder JS, Boo SZ, et al. Intracoronary ultrasound in cardiac transplant recipients: in vivo evidence of angiographically silent intimal thickening. Circulation 1992;85:979-87.

7 Rickenbacher PR, Pinto FJ, Chenzbraun A, Botas J, Lewis NP, Alderman $\mathrm{EZ}$, et al. Incidence and severity of transplant coronary artery disease early and up to 15 years after transplantation as detected by intravascular ultrasound. $\mathcal{A} \mathrm{Am}$ Coll Cardiol 1995;25:171-7.

8 Yeung AC, Davis SF, Hauptman PJ, Kobashigawa JA, Miller LW, Valantine $\mathrm{HA}$, et al. Incidence and progression of transplant coronary artery disease over 1 year: results of a multicenter trial with use of intravascular ultrasound. $f$ Heart Lung Transplant 1995; 14:S215-20.

9 Fish RD, Nabel Selwyn AP, Ludmer PL, Mudge GH, Kirshenbaum JM, et al. Responses of coronary arteries of cardiac transplant patients to acetylcholine. $\mathcal{F}$ Clin Invest 1988;81:21-31.

10 Anderson TJ, Meridith IT, Uehata A, Mudge GH, Selwyn AP, Gauz P, et al. Functional significance of intimal thickening as detected by intravascular ultrasound early and late after cardiac transplantation. Circulation 1993;88:1093-100.

11 Treasure CB, Vita JA, Ganz P, Ryan JCTS, Schoen FJ, Vekshtein VI, et al. Loss of coronary microvascular response to acetylcholine in cardiac transplant patients. Circulation 1992;86:1156-64.

12 Meiser BM, Wenke K, Theiry J, Brandl U, Mair H, Kur F, et al. Prevention and treatment of graft vessel disease after heart transplantation. Transplant Proc 1995;27:1931-5.

13 Kobashigawa JA, Katznelson S, Laks H, Johnson JA, Yeatman L, Wang $\mathrm{XM}$, et al. Effect of pravastatin on outcomes after cardiac transplantation. $N$ Engl f Med 1995;333:621-7.

14 Halle AA, DiSciascio G, Massin EK, Wilson RF, Johnson MR, Sullivan $\mathrm{HJ}$, et al. Coronary angioplasty, atherectomy and bypass surgery in cardiac transplant patients. $\mathcal{F} \mathrm{Am}$ Coll Cardiol 1995;26:120-8.

15 Ensley RD, Hunt S, Taylor DO, Renlund DG, Menlove RL, Karwande SV, et al. for the Registry of the International Society for Heart and Lung -Transplantation, and contributing investigators. Predictors of survival - Transplantation, and contributing investigators. Predictors of survival 1992;11:\$142-58. 